\title{
BMJ Open Quality Improving critical incident reporting in primary care through education and involvement
}

\author{
Beate Sigrid Müller, ${ }^{\oplus 1}$ Martin Beyer, ${ }^{1}$ Tatjana Blazejewski, ${ }^{1}$ Dania Gruber, ${ }^{1}$ \\ Hardy Müller, ${ }^{2}$ Ferdinand Michael Gerlach ${ }^{1}$
}

To cite: Müller BS, Beyer M, Blazejewski T, et al. Improving critical incident reporting in primary care through education and involvement. BMJ Open Quality 2019;8:e000556. doi:10.1136/ bmjoq-2018-000556

Received 18 October 2018 Revised 10 July 2019 Accepted 31 July 2019
Check for updates

(C) Author(s) (or their employer(s)) 2019. Re-use permitted under CC BY-NC. No commercial re-use. See rights and permissions. Published by BMJ.

${ }^{1}$ Institute of General Practice, Goethe University Frankfurt, Frankfurt am Main, Germany ${ }^{2}$ Techniker Krankenkasse, Hamburg, Germany

Correspondence to Dr Beate Sigrid Müller, Institute of General Practice, Goethe University Frankfurt, Frankfurt am Main 60590, Germany; b.mueller@allgemeinmedizin. uni-frankfurt.de

\section{ABSTRACT}

Background Critical incident reporting systems (CIRS) can be an important tool for the identification of organisational safety needs and thus to improve patient safety. In German primary care, CIRS use is obligatory but remains rare. Studies on CIRS implementation in primary care are lacking, but those from secondary care recommend involving management personnel.

Objective This project aimed to increase CIRS use in 69 practices belonging to a local practice network.

Methods The intervention consisted of the provision of a web-based CIRS, accompanying measures to train practice teams in error management and CIRS, and the involvement of the network's management. Three measurements were used: (1) number of incident reports and user access rates to the web-based CIRS were recorded, (2) staff were given a questionnaire addressing incident reporting, error management and safety climate and (3) qualitative reflection conferences were held with network management.

Results Over 20 months, 17 critical incidents were reported to the web-based CIRS. The number of staff intending to report the next incident online decreased from $42 \%$ to $20 \%$ of participants. In contrast, the number of practices using an offline CIRS (eg, incident book) increased from $23 \%$ to $49 \%$ of practices. Practices also began proactively approaching network management for help with incidents. After project completion, participants scored higher in the patient safety climate factor 'perception of causes of errors'. For many practices, the project provided the first contact with structured error management.

Conclusion Specific measures to improve the use of CIRS in primary care should focus on network management and practice owners. Practices need basic training on safety culture and error management. Continuing, practices should implement an offline CIRS, before they can profit from the exchange of reports via web-based CIRS. It is crucial that practices receive feedback on incidents, and trained network management personnel can provide such support.

\section{PROBLEM}

Patient safety incidents frequently occur in primary care and many such events are considered avoidable. Critical incident reporting systems (CIRS) can be an important tool for the identification of organisational safety needs. ${ }^{1}$ In German primary care, CIRS are obligatory by law but rarely used..$^{2-4}$ We therefore decided in cooperation with a statutory health insurance company to initiate a quality improvement project to encourage CIRS use in primary care. Studies from secondary care recommend integrating management personnel in CIRS implementation procedures. ${ }^{56}$ In the average German practice, the physician owner of the practice fulfils all management duties in addition to daily patient care. We therefore decided to conduct this study in a practice network with designated network management and more resources available to support the project.

A practice network in Nuremberg, Germany, was chosen to be the study site. In order to improve care through collaboration, 69 practices of different disciplines had joined this network, for example, general practitioners (GPs), ophthalmologists, internists, gynaecologists and orthopaedic surgeons. As GPs have no gatekeeper function in Germany, patients can seek direct access to specialists. Therefore, specialist physicians in German ambulatory care may be involved in both primary and secondary care. In these network practices, around 130 physicians and 400 healthcare assistants (HCAs) cared for 100000 patients per quarter. Network management consisted of three executive board members (physicians) and five employees at the network office (HCAs and business economists). At the time the project was launched, practices dealt with critical incidents individually, and neither a CIRS nor standards regulating the management of incidents yet existed.

\section{BACKGROUND}

In preparation for this project, we conducted a literature search on interventions designed to increase incident reporting in healthcare. Robust studies scarcely existed for secondary care settings, and a Cochrane Review on those that were available was unable 'to draw 
conclusions for clinical practice' due to study limitations. ${ }^{7}$ Even fewer studies on introducing a CIRS or improving an established system had been conducted in primary healthcare.$^{8-10}$ As a result, we decided to conduct a quality improvement project that consisted of educating and training practice teams to introduce a new web-based reporting system and the participation of network management.

\section{MEASUREMENT}

When we introduced the new CIRS, no critical incidents had yet been reported.

During the course of the project, we planned several quantitative and qualitative assessments.

1 . The number of reported incidents and user access rates were recorded anonymously.

2. A staff questionnaire was sent out to practices at the beginning and end of the project. It addressed attitudes towards incident reporting, current error management procedures and the views of practice personnel on several aspects of safety climate. The items were based on the "theory of planned behaviour"11 12 and the 'Frankfurt patient safety climate questionnaire for general practices'. ${ }^{13}$ We used the questionnaire to detect changes in practice teams' attitudes and processes (for details see Bauer et al). ${ }^{14}$

3. Qualitative reflection conferences were held with the network's management every $4-6$ months to evaluate how the project was developing.

\section{DESIGN}

First, a project plan was developed by both the project team (authors MB, TB, BM, DG) and network management. Beginning in June 2015, an intervention was agreed on that consisted of the provision of a web-based incident reporting system and accompanying measures over a period of 20 months.

The established reporting system was similar to the reporting system (www.jeder-fehler-zaehlt.de) for general practices, ${ }^{3}$ except that access was granted only to network practice team members. The system consisted of a general welcome page with information on the project, a report entry form, a discussion forum that followed each report and information materials on incident prevention in practices. Reporting was anonymous, whereas participants in the discussion forum used their real names.

As suggested in similar studies, training workshops were held for practice team members, and information provided on how to use and access the incident reporting system. ${ }^{15}$ They took place on several occasions, so that each team member had the chance to participate. Slightly different workshops were carried out for physicians and HCAs.

In order to foster communication about the project and the reporting system, presentations on best practice procedures relating to incident management took place at regular network meetings. Furthermore, newsletters providing information on the latest incident reports and calling for new incident reports on a variety of topics were sent to practices via e-mail. Both measures had previously been used to improve incident reporting in other studies. ${ }^{91617}$ Over the course of the project, we also carried out focus group discussions with physicians and HCAs to identify improvement opportunities (for details see Gruber $e t a l) .{ }^{18}$

\section{STRATEGY}

Plan, do, study, act (PDSA) cycle 1 (project month 1)

Our first intervention involved discussing the wording used to describe incidents with key stakeholders, as common terminology was considered important in the literature on CIRS implementation. ${ }^{8}{ }^{19}$ Several terms, for example, for 'critical incident' and 'error management', were discussed on the basis of commonly used terminology. ${ }^{20}$ It was important to network management and the other key stakeholders that the word 'error' was not used, as practices might associate it with blame and punishment. The parties agreed to use 'critical incident' rather than 'error', and 'risk management' rather than 'error management'. We updated all text passages in the web-based CIRS accordingly before moving on to PDSA cycle 2.

\section{PDSA cycle 2 (project months 1-4)}

The second intervention involved providing every practice team member with log-in-data to the web-based CIRS and the opportunity to participate in training workshops. Two of the network physicians were trained to moderate the discussion forum of the CIRS. These measures were undertaken during the same 4-month period to ensure participants could make use of their accounts immediately.

\section{PDSA cycle 3 (project months 6,8 and 12)}

In a next step, practice teams were reminded to use the CIRS during three network meetings (in the presence of both physicians and HCAs). To achieve this, the physician moderators (see the PDSA cycle 2 section) presented incident reports and pointed out the advantages of structured error management. The majority of practices was present at these network meetings. In order to reach those who were not, we initiated PDSA cycle 4.

\section{PDSA cycle 4 (project months 9-11)}

At an interval of 8 weeks, two newsletters were sent to all practice staff registered in the web-based CIRS via email. They each addressed one of the latest incident reports and asked for reports on one specific topic (first newsletter: interface between practices and second newsletter: communication within the practice team). In the same months, the respective topic was also presented and discussed at network meetings. A third newsletter on medication safety was planned but not sent out, due to the practice network's tight schedule. When user access rates of the web-based CIRS declined, we decided to move on to PDSA cycle 5. 


\section{PDSA cycle 5 (project month 16)}

The fifth intervention involved conducting focus groups with 11 physicians and five HCAs to find out about barriers and facilitators to CIRS use in order to improve reporting rates. Discussions revealed several important points, for example, that the web-based reporting form contained some barriers itself. As a result, we reduced the number of text entry fields to accelerate the reporting process. During discussions, it also became clear that several practice owners did not want their HCAs to file reports to the web-based CIRS. They stated HCAs would probably describe the incident incorrectly. ${ }^{18}$ These findings were the reason for PDSA cycle 6 , which we started in order to further improve the safety culture in the participating practices.

\section{PDSA cycle 6 (project month 17)}

A presentation on risk management and safety culture in aviation and healthcare was given to network physicians and network management. On the suggestion of the network management, a physician (with a pilot's license) was chosen for this presentation to address the network physicians on peer level.

\section{PDSA cycle 7 (project month 20)}

In the course of the project, rather than reporting and discussing cases in the web-based CIRS, practices turned increasingly to network management for help analysing incidents. We therefore decided to hold a train-thetrainer workshop for management personnel and to train them in incident analysis. Management regarded this workshop as extremely useful in their daily communication with practices.

\section{Patient and public involvement}

Patients and the public were not involved in the development or implementation of the project.

\section{RESULTS}

The introduction of an online critical incident reporting system in 69 practices revealed several points of crucial relevance to the further implementation of CIRS in (German) primary care. The three measurement methods revealed the following results.

\section{Number of reported incidents and user access rates}

Over the course of the project, 17 critical incidents were reported to the web-based CIRS. Access rates were high at the beginning of the project but decreased over time (see figure 1). Continuous reminders (PDSA cycles 3 and 4, months 6-11) appeared to be important to encourage participants to continue to report.

\section{Staff questionnaire about error management and safety climate}

The number of staff intending to report the next incident to the web-based CIRS decreased from $42 \%$ to $20 \%$ of participants during the course of the project. Interestingly, however, the number of practices using an offline CIRS (eg, incident book) increased from $23 \%$ to $49 \%$ of practices. After the project, participants also scored better in the patient safety climate factor 'perception of causes of errors'.

\section{Reflection of network management}

Network management regularly discussed the development of the project. From their perspective, the practice teams changed their attitudes in favour of a culture in which errors are discussed openly. While practices were at first rather cautious, they proactively and increasingly approached network management for help with critical incidents during the course of the project. Network management therefore asked the project team for a train-the-trainer workshop to enable them to provide coaching and feedback that was tailored to suit the practice concerned. Management personnel also identified barriers to the success of the project that they recognised during meetings with practices. Hierarchical structures and practice owners that viewed the project as useless and a waste of time were seen as hindering the successful implementation of structured error management.

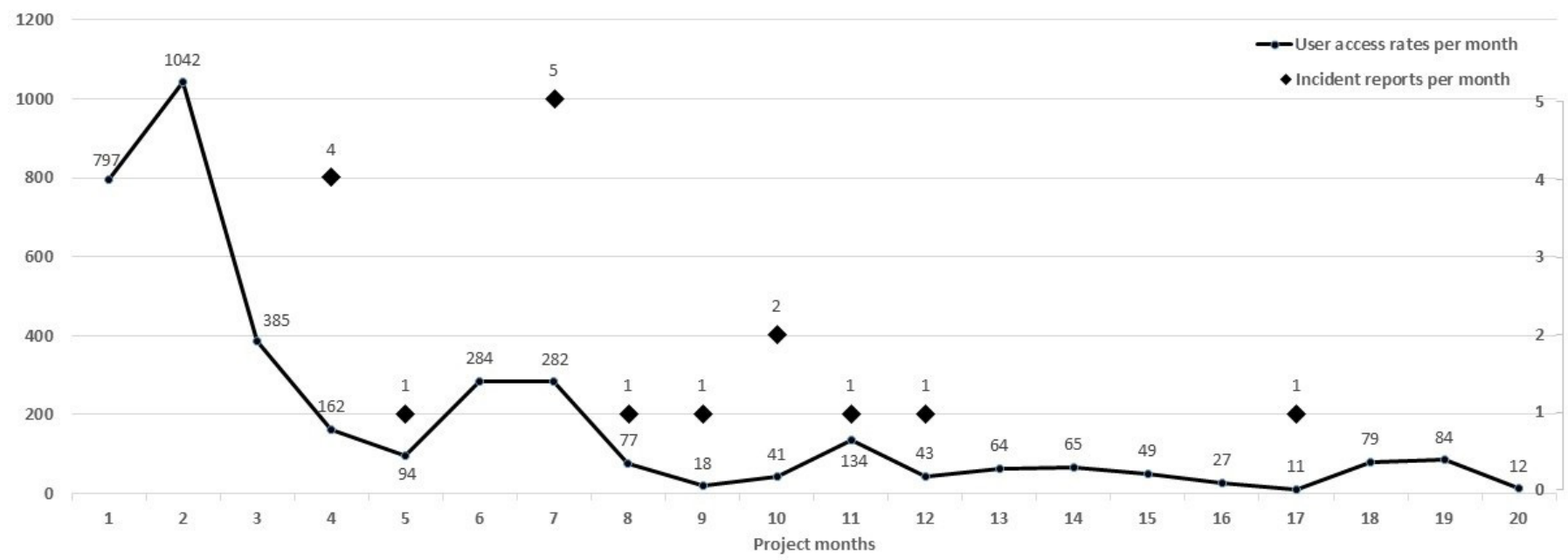

Figure 1 User access rates and incident reports per month. 


\section{LESSONS AND LIMITATIONS}

We learnt many important lessons during the course of our project. Error management in German ambulatory practices is still on a low level but can be improved by specific measures. Workshops on human factors and system theory ${ }^{21}$ should be used to improve the general understanding of safety culture, error management and CIRS. Our practices obviously supported the idea of CIRS, but not of the web-based form. We therefore recommend for practices to start with an offline CIRS and in a second step to exchange reports via a web-based CIRS. Furthermore, feedback to individual practices on their critical incidents is crucial. We had hoped practices would provide each other with feedback in the discussion forum of our web-based CIRS. Instead, practices turned to network management for help when something went wrong. We therefore changed our project plan and held a train-the-trainer workshop for management personnel to enable them to support practices better (PDSA cycle 7). Management considered this extremely helpful. As we had expected, network management played a central role as a facilitator of error management within the practice network. Nevertheless, we would recommend that subsequent studies focus equally on practice owners, as the main challenge we faced was the practice owners' attitudes towards error management and CIRS. Ultimately, they decide on who reports what in their practice and on changes to practice structures and not the network's management.

Several limitations should be taken into account. The practice network participated voluntarily and had already undertaken several quality improvement projects in other fields. Thus, network management and practices were probably more familiar with quality improvement than most practice networks, which limits generalisability. Unfortunately, our project ended shortly after the trainthe-trainer workshop. More research is needed here to investigate whether coaching and feedback by network management is feasible and accepted by practices.

\section{CONCLUSION}

Network management and practice teams dealt intensively with error management and CIRS during this project. For many practices, it was probably their first close contact with structured error management. Practice teams' reservations regarding error management declined in the course of the project and offline CIRS were increasingly introduced. Nevertheless, the web-based CIRS was not (yet) used regularly.

Acknowledgements The authors would like to express their thanks to all participating practices and to the network management personnel. We also thank Phillip Elliott for the language review of the paper.

Contributors BSM, MB, TB, HM and FMG contributed to the conception and design of the project and critical revision of the article and final approval of the version to be published. BSM, MB, TB and DG contributed to the project plan, data collection, data analysis and interpretation. BSM wrote the first draft of the article.

Funding This study was funded by Techniker Krankenkasse.
Competing interests BSM, MB, TB, DG and FMG received grants from the German Statutory Health Insurance Company Techniker Krankenkasse (TK) for the study. HM is an employee of TK and General Secretary of the German Coalition for Patient Safety (APS).

Patient consent for publication Not required.

Provenance and peer review Not commissioned; externally peer reviewed.

Open access This is an open access article distributed in accordance with the Creative Commons Attribution Non Commercial (CC BY-NC 4.0) license, which permits others to distribute, remix, adapt, build upon this work non-commercially, and license their derivative works on different terms, provided the original work is properly cited, appropriate credit is given, any changes made indicated, and the use is non-commercial. See: http://creativecommons.org/licenses/by-nc/4.0/.

\section{REFERENCES}

1. Howell A-M, Burns EM, Hull L, et al. International recommendations for national patient safety incident reporting systems: an expert Delphi consensus-building process. BMJ Qual Saf 2017;26:150-63.

2. Bundesausschuss G. Richtlinie des Gemeinsamen Bundesausschusses über grundsätzliche Anforderungen an ein einrichtungsinternes Qualitätsmanagement für Vertragsärztinnen und Vertragsärzte, Vertragspsychotherapeutinnen und Vertragspsychotherapeuten. medizinische Versorgungszentren, Vertragszahnärztinnen und Vertragszahnärzte sowie zugelassene Krankenhäuser: Qualitätsmanagement-Richtlinie/QM-RL, 2015.

3. Beyer M, Blazejewski T, Güthlin C, et al. Das hausärztliche Fehlerberichts- und Lernsystem 'jeder-fehler-zaehlt.de' Berichtsbestand und Nutzungsperspektiven: jeder-fehler-zaehlt.de Content of and prospective benefits from a critical incident reporting and learning system (CIRS) for primary care. Zeitschrift für Evidenz, Fortbildung und Qualität im Gesundheitswesen 2015;109:62-8.

4. Rea D, Griffiths S. Patient safety in primary care: incident reporting and significant event reviews in British general practice. Health Soc Care Community 2016;24:411-9.

5. Dowell L. Implementing a patient safety team to reduce serious incidents. BMJ Qual Improv Report 2013;2.

6. Smith A, Hatoun J, Moses J. Increasing trainee reporting of adverse events with monthly Trainee-Directed review of adverse events. Acad Pediatr 2017;17:902-6.

7. Parmelli E, Flodgren G, Fraser Scott G, et al. Interventions to increase clinical incident reporting in health care(8. John Wiley \& Sons, Ltd, 2012.

8. Donnelly P. Improving reporting of critical incidents through education and involvement. BMJ Qual Improv Report 2015;4.

9. Crane S, Sloane PD, Elder N, et al. Reporting and using near-miss events to improve patient safety in diverse primary care practices: a collaborative approach to learning from our mistakes. J Am Board Fam Med 2015;28:452-60.

10. Verbakel NJ, Langelaan M, Verheij TJM, et al. Effects of patient safety culture interventions on incident reporting in general practice: a cluster randomised trial. British Journal of General Practice 2015;65:e319-29.

11. Ajzen I. The theory of planned behavior: habit, perceived control, and reasoned action. Mannheim: Mannheimer Zentrum für Europäische Sozialforschung, 2000.

12. Mascherek $A C$, Gehring $K$, Bezzola $P$, et al. Using the theory of planned behaviour to model antecedents of surgical checklist use: a cross-sectional study. BMC Health Serv Res 2015;15.

13. Hoffmann B, Domanska OM, Albay Z, et al. The Frankfurt patient safety climate questionnaire for general practices (FraSiK): analysis of psychometric properties. BMJ Qual Saf 2011;20:797-805.

14. Bauer A, Beyer M, Paulitsch M, et al. Steigerung Der Nutzung von Fehlerberichts- und Lernsystemen: eine Fragebogenstudie: increased utilization of incident reporting systems - a questionnaire survey. Zeitschrift für Allgemeinmedizin 2018;94:70-5.

15. Lederman R, Dreyfus S, Matchan J, et al. Electronic error-reporting systems: a case study into the impact on nurse reporting of medical errors. Nurs Outlook 2013;61:417-26.

16. Stewart DA, Junn J, Adams MA, et al. House staff participation in patient safety reporting: identification of predominant barriers and implementation of a pilot program. South Med J 2016;109:395-400.

17. Koining J, Stelzl L. Die Initiative PatientInnensicherheit Steiermark (IPS) - ein Netzwerk entsteht. Qualitas 2014;2.

18. Gruber D, Blazejewski T, Beyer M, et al. Fehlermanagement in Der ambulanten praxis. Fokusgruppen MIT Ärztinnen, Ärzten und Medizinischen Fachangestellten: error management in outpatient 
settings. Focus Groups Involving Physicians and Medical Assistants. Zeitschrift für Allgemeinmedizin 2018;94:110-5.

19. Kusano AS, Nyflot MJ, Zeng J, et al. Measurable improvement in patient safety culture: a Departmental experience with incident learning. Pract Radiat Oncol 2015;5:e229-37.
20. Barth S, Immel-Sehr A. Reden IST gold: Kommunikation nACh einem Zwischenfall. 1st edn. Bonn: Aktionsbündnis Patientensicherheit, 2011.

21. Reason JT. Human error. Cambridge, UK: Cambridge Univ. Press, 1990. 